\title{
Experimental hydrodynamics imaging and undulatory movement equation of steady swimming fish (Pangasius sanitwongsei)
}

\author{
M. Abbaspour \& A. S. Vaghefi \\ Department of Mechanical Engineering, \\ Science and Research Branch, Islamic Azad University, Tehran, Iran
}

\begin{abstract}
This paper presents a quantitative characterization of caudal fin. Steady swimming of Pangasius sanitwongsei was studied experimentally and taped by high speed digital video and undulatory movement of fish was revealed. As a result, an experimental equation was found. This equation indicates that, as much as the ratio of the end part of fish with high undulatory movement (body and caudal fin) to the total length goes up, the ratio of amplitude to the total length increases. Consequently, displacement increases and thrust force increases too. Then undulatory movement equation of fish swimming was calculated by fitting a second order function which describes wave amplitude of this type of fish. All the findings in these researches could be applied to design a robotic fish.

Keywords: undulatory movement equation, fish swimming, robotic fish, video image, Pangasius.
\end{abstract}

\section{Introduction}

For a long time, scientists have marveled at the graceful way in which fish swim, turn and accelerate [1]. In nature, fish are propelled by the undulatory motion of their body and have gained wonderful swimming ability over the thousands of years of evolution. Tuna swim with high speed and high efficiency. Pike accelerate in a flash, and eels can swim skillfully into narrow holes [2]. From a mechanics appearance, their movements raise questions; how good they are as 
swimmers? Are they more efficient than a propeller driven under water vehicle? [1]. Many researchers have been trying to answer these questions. Fish swim mode has been studied extensively using experimental, theoretical and numerical techniques. Experimental studies have been applied on the real fish, robot fish and biomimetic foils.

In 1994, the first robot fish named robotuna was developed at MIT [3]. In 1998, Anderson et al. studied the flow around two-dimensional flapping foils as a function of the angle of attack and the Strouhal number. The Strouhal number is defined as $\mathrm{St}=\mathrm{f} . \mathrm{A} / \mathrm{u}$, where $\mathrm{f}$ denotes the frequency of foil oscillation, A denotes the characteristic width of the created jet flow and $u$ is the speed of the foil. They presented wake patterns as a function of the Strouhal number and the angle of attack [4]. In 2002, Lauder et al. studied hydrodynamics of fish movement with Digital Particle Image Velocimetry (DPIV) which allowed empirical analysis of force magnitude and direction. They examined fin function in four ray finned fish clades; sturgeon, trout, sunfish and mackerel [6]. In 2004, Gilbert characterized quantitatively the complex three dimensional kinematics of pectoral fin swimming in bluegill sunfish. He taped fish swimming at several speeds in a flow tank that is $45 \mathrm{~cm}$ long by $18 \mathrm{~cm}$ wide and $18 \mathrm{~cm}$ high. Flow patterns were visualized with the high speed (500fps) and high resolution (1024 x 1024 pixels) camera [5]. In 2007, Standen and Lauder presented that the dorsal fins produce vortices that are encountered by the caudal fin. These vortices can increase the thrust produced by caudal fins [7].

In this study, the flow patterns of caudal fin locomotion were visualized and the kinematic parameters of Pangasius sanitwongsei were determined which in turn ended up defining the equation of fish swimming.

\section{Fish swimming mode}

Several design characteristics in fish are useful to design, robotic devices for propulsion purposes in underwater vehicles. One of the main characteristics is swimming mode [8]. One way of considering fish locomotion is to classify fish into groups based on fin use. Those fish swim with their median fins (dorsal and anal) and paired fins (pectoral and pelvic) are termed MPF (median and paired fin) swimmers and those fish use primarily their body and caudal fin are classified as BCF (body and caudal fin) swimmers (fig. 1) [9].

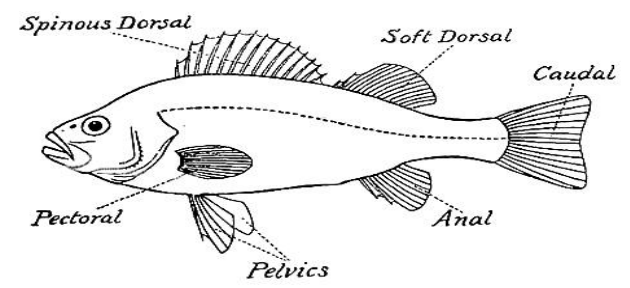

Figure 1: Illustrates median and paired fins. 
MPF is used at slow speeds, to increase the maneuverability and propulsive efficiency while BCF movement can increase thrust force and accelerations [8]. Lindsey classifies the different modes of propulsive movements of fish into four groups [10]: anguiliform, subcarangiform, carangiform and thunniform (fig. 2).

Nonetheless, the aim here is to examine the BCF mechanism of Pangasius sanitwongsei.
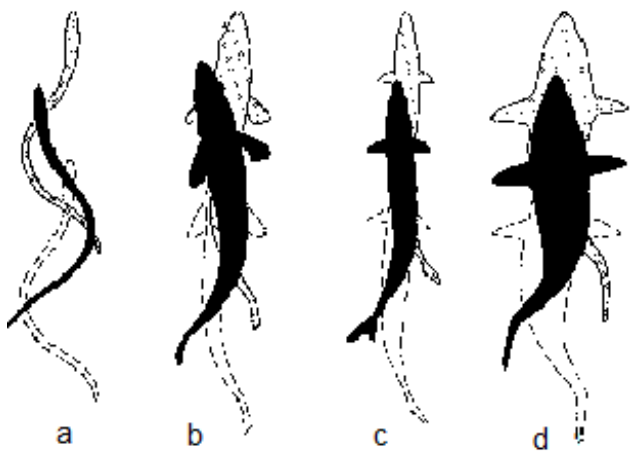

Figure 2: $\quad$ Different modes of propulsive movements of fish by Lindsey [10]; (a) anguilliform, (b) subcarangiform, (c) carangiform and (d) thunniform. In anguiliform mode, the whole body influences on thrust force. In subcarangifom mode, the posterior half of the body participates in propulsive mechanism. For carangiform mode, the last third of the body length has got an undulatory movement. In thunniform mode, thrust is generated by tail movement.

\section{Preliminary observation}

There are several important non- dimensional numbers in fish swimming. In 1988, Webb defined three main factors which participate in fish swimming; Reynolds number, Strouhal number and shape [11].

The Reynolds number is the ratio of inertial forces over viscous forces, and is defined as $R e=u . L / v$, where $u$ is the velocity of fish swimming, $L$ is the length of the fish body and $v$ is kinematic viscosity of water. Another non-dimensional parameter which participates in fish movement, is Strouhal number defined as $\mathrm{St}=\mathrm{f} . \mathrm{A} / \mathrm{u}$, where $\mathrm{f}$ is the tail-beat frequency, $\mathrm{A}$ is the amplitude of undulatory movement of tail and $u$ is the average velocity of fish $[1,8,11]$. The Strouhal number is the ratio of unsteady to inertial force [13]. Another commonly used non-dimensional parameter is $\mathrm{Lf} / \mathrm{L}$. In this work, $\mathrm{Lf} / \mathrm{L}$ was chosen as a characteristic of shape, where Lf was the distance from top of the head to end of the head. This is a major point and displays less variation of head to the more variation of the body and caudal fin. In fact, it is a fixed point in the fish body. 
On the other hand, $\mathrm{L}$ is the length of the fish body, the major deformable length of the body (responsible for generating thrust) is L-Lf in the rear and the position $\mathrm{x}=$ Lf gives the minimum amplitude along the entire body (fig. 3) [12].

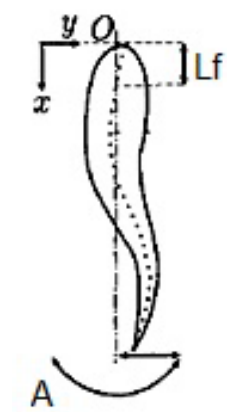

Figure 3: $\quad$ Shows Lf in typical fish movement [12].

Pangasius sanitwongsei (Fig. 4) with several total lengths was studied in a glassy tank that was $90 \mathrm{~cm}$ long by $30 \mathrm{~cm}$ wide and $45 \mathrm{~cm}$ high. The water was at $20^{\circ \mathrm{c}}$ and kinematic viscosity was $v=1.005 \times 10^{-6}\left(\mathrm{~m}^{2} / \mathrm{s}\right)$. As mentioned before, one of the main factors participating in fish swimming, is the shape. In this work, $\mathrm{Lf} / \mathrm{L}$ was chosen as a characteristic of shape. Therefore, several fish with different body length were chosen; $12 \mathrm{~cm}, 9 \mathrm{~cm}, 7.5 \mathrm{~cm}$ and $5.8 \mathrm{~cm}$, with $\mathrm{Lf} / \mathrm{L}$ $0.250,0.280,0.270$ and 0.260 respectively.

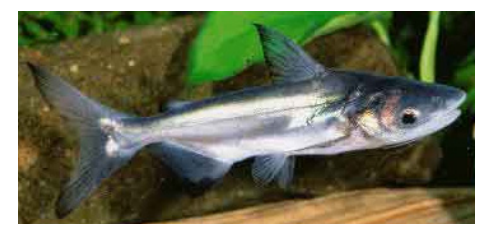

Figure 4: Pangasius sanitwongsei was studied in this work.

To evaluate fish swimming behavior, the images were videotaped by a cube 3 camera at $2500 \mathrm{fps}$ at maximum resolution, maximum frame capture rate of 120,000 fps.

Figure 5 summarizes the experimental apparatus employed in this study. A planar slice of the flow was illuminated with a $100 \mathrm{~mW}$ laser. Flow pattern was seeded with polystyrene small particles (40-60micro meter). Fish swimming was visualized several times for each fish and finally some suitable frames were chosen. Figure 6 shows five frames of Pangasius sanitwongsei with $9 \mathrm{~cm}$ body length and $\Delta \mathrm{t}=20 \mathrm{msec}$. 


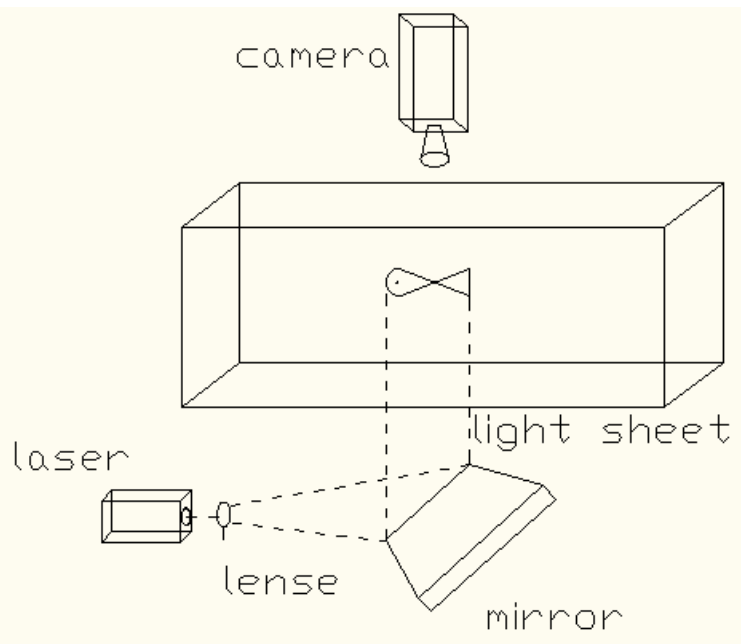

Figure 5: Schematic of the experimental set up. Images of fish swimming were obtained with the high speed video camera. A planar slice of the flow was illuminated with a $100 \mathrm{~mW} \mathrm{Nd}$.Yag laser $\left(\lambda=532^{\mathrm{nm}}\right)$ aimed at the $45^{\circ}$ front-surface mirror positioned below the flow tank.
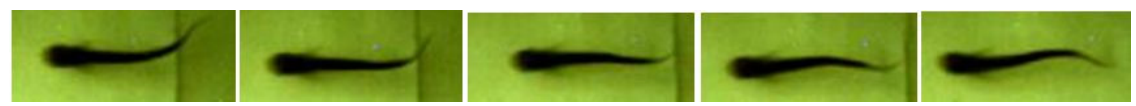

Figure 6: Five original images of Pangasius sanitwongsei by $\mathrm{L}=9 \mathrm{~cm}$ at $\Delta \mathrm{t}=0.02 \mathrm{~s}$. Undulatory movement of caudal fin has been presented. Half of wavelength has been represented at $80 \mathrm{msec}$.

\section{Results}

In this experimental study, undulatory movement of Pangasius sanitwongsei was observed and taped. Among all video images, two suitable captures were chosen for each case. Figure 6, displays five sequential images of Pangasius sanitwongsei by total length $9 \mathrm{~cm}$ at $\Delta \mathrm{t}=20 \mathrm{msec}$. These images reveal undulatory movement of fish tail while, it is passing half of the wave length. According to these video images, frequency, amplitude and velocity of fish swimming were obtained. Then, two dimensionless parameters, Reynolds number and Strouhal number were found. Table 1 shows a summary of fish swimming variables of Pangasius sanitwongsei by total length $9 \mathrm{~cm}$. These parameters were obtained from experimental results.

According to the video images, patterns of body undulatory movement of Pangasius sanitwongsei were drawn. Figure 7, shows body outlines taken from movements of fish swimming of Pangasius sanitwongsei with total length, 
Table 1: $\quad$ Summary of fish swimming variables of Pangasius sanitwongsei. All measurements tabulated from experimental results of Trout and Pangasius sanitwongsei. A displays amplitude, $f$ displays frequency and $\mathrm{u}$ displays velocity. Velocity and amplitude have been shown as a proportion of total length.

\begin{tabular}{|c|c|c|c|c|c|c|c|c|}
\hline Table 2 & $\mathrm{~L}(\mathrm{~cm})$ & $\operatorname{Lf}(\mathrm{cm})$ & $\mathrm{A}(\mathrm{cm})$ & $f(1 / s)$ & $\mathrm{u}(\mathrm{cm} / \mathrm{s})$ & $\mathrm{Lf} / \mathrm{L}$ & $\mathrm{St}$ & $\operatorname{Re}$ \\
\hline \multirow[t]{3}{*}{ No.1 } & \multirow[t]{3}{*}{12} & \multirow[t]{3}{*}{3.00} & $0.28 \mathrm{~L}$ & 1.67 & $0.98 \mathrm{LT}^{-1}$ & \multirow[t]{3}{*}{0.250} & 0.47 & 14042 \\
\hline & & & $0.19 \mathrm{~L}$ & 1.43 & $0.81 \mathrm{LT}^{-1}$ & & 0.33 & 11678 \\
\hline & & & $0.22 \mathrm{~L}$ & 1.67 & $0.89 \mathrm{LT}^{-1}$ & & 0.41 & 12800 \\
\hline \multirow[t]{3}{*}{ No.2 } & \multirow[t]{3}{*}{9} & \multirow[t]{3}{*}{2.52} & $0.11 \mathrm{~L}$ & 8.33 & $5.38 \mathrm{LT}^{-1}$ & \multirow[t]{3}{*}{0.280} & 0.17 & 43397 \\
\hline & & & $0.33 \mathrm{~L}$ & 6.25 & $5.67 \mathrm{LT}^{-1}$ & & 0.36 & 45672 \\
\hline & & & $0.13 \mathrm{~L}$ & 8.33 & $5.37 \mathrm{LT}^{-1}$ & & 0.21 & 43379 \\
\hline \multirow[t]{3}{*}{ No.3 } & \multirow[t]{3}{*}{7.5} & \multirow[t]{3}{*}{2.03} & $0.12 \mathrm{~L}$ & 5.00 & $2.60 \mathrm{LT}^{-1}$ & \multirow[t]{3}{*}{0.270} & 0.25 & 14366 \\
\hline & & & $0.15 \mathrm{~L}$ & 8.33 & $3.46 \mathrm{LT}^{-1}$ & & 0.35 & 19343 \\
\hline & & & $0.13 \mathrm{~L}$ & 5.00 & $3.22 \mathrm{LT}^{-1}$ & & 0.21 & 18037 \\
\hline \multirow[t]{3}{*}{ No.4 } & \multirow[t]{3}{*}{5.8} & \multirow[t]{3}{*}{1.51} & $0.23 \mathrm{~L}$ & 3.12 & $1.96 \mathrm{LT}^{-1}$ & \multirow[t]{3}{*}{0.260} & 0.37 & 6568 \\
\hline & & & $0.31 \mathrm{~L}$ & 4.17 & $2.85 \mathrm{LT}^{-1}$ & & 0.46 & 9569 \\
\hline & & & $0.20 \mathrm{~L}$ & 3.55 & $2.15 \mathrm{LT}^{-1}$ & & 0.33 & 7260 \\
\hline
\end{tabular}

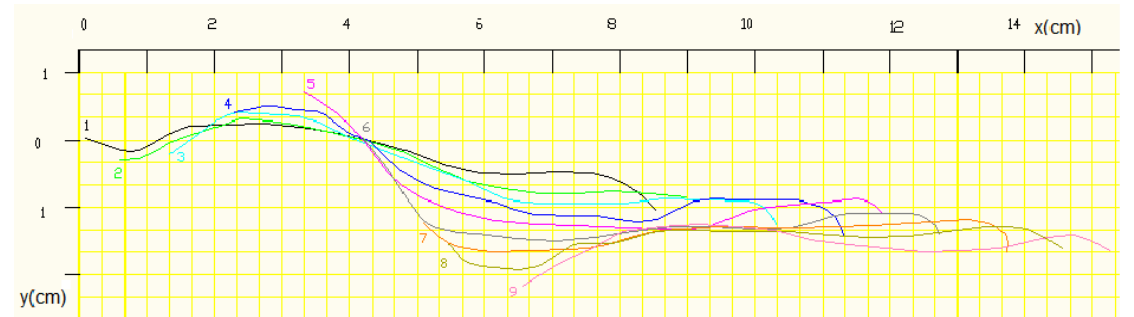

Figure 7: Patterns of body undulatory movement of Pangasius sanitwongsei by $\mathrm{L}=9 \mathrm{~cm}$ at $\Delta \mathrm{t}=20 \mathrm{~m} \mathrm{sec}$.

$\mathrm{L}=9 \mathrm{~cm}$. This image displays one tail-beat cycle recorded at intervals of $20 \mathrm{~ms}$. As this figure illustrates fish swimming variables such as amplitude, frequency and velocity could be determined. In addition, this figure represents that the 
amplitude of this wave increases dramatically near the tail while it is very low near the head.

Figure 8 shows the undulatory movement of caudal fin of four Pangasius sanitwongsei in steady swimming. The time interval between sequential plotted tail positions is $20 \mathrm{~m} \mathrm{sec}$. The total experimental recording time is $200 \mathrm{~m} \mathrm{sec}$. This figure illustrates that undulatory movement of caudal fin of Pangasius sanitwongsei is similar to a sinusoidal form. Furthermore, it could be understood that undulatory movement of caudal fin becomes closer to a complete sinusoidal form, as $\mathrm{Lf} / \mathrm{L}$ increases. In order to clarify this, undulatory movements of caudal fin of four Pangasius sanitwongsei with different $\mathrm{Lf} / \mathrm{L}, 0.250,0.260,0.270$ and 0.280 were studied. As shown in figure 8 , even though the capture time for lower $\mathrm{Lf} / \mathrm{L}$ is almost three times of the case of higher $\mathrm{Lf} / \mathrm{L}$, its behavior is not in a sinusoidal form.

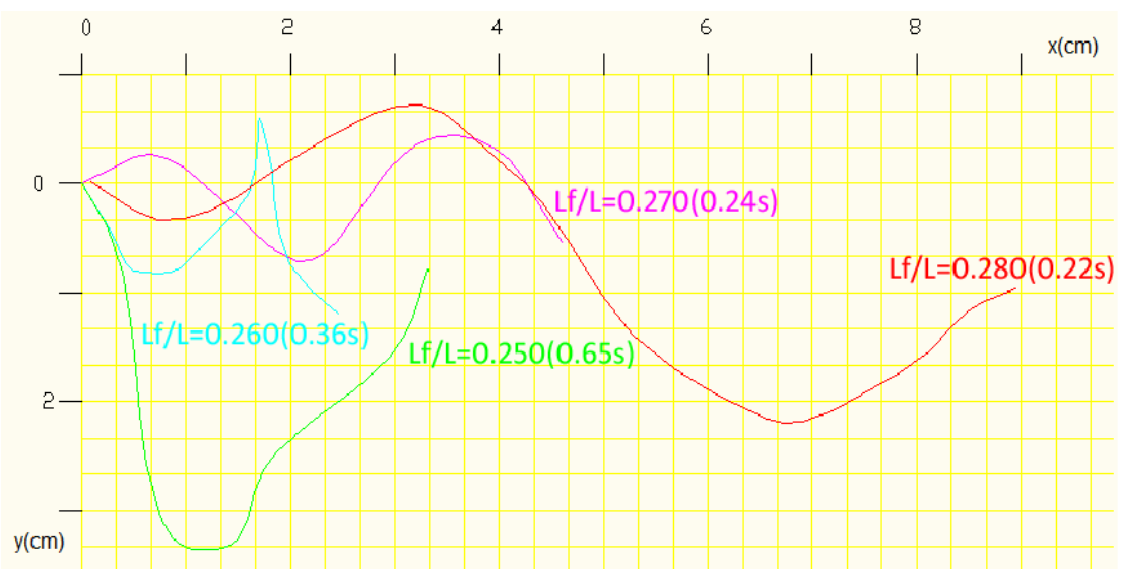

Figure 8: Undulatory movement of caudal fin of four Pangasius sanitwongsei with different $\mathrm{Lf} / \mathrm{L}$. The number in parenthesis displays total experimental recording time for each fish.

\section{Discussion}

In this experimental trial, Pangasius sanitwongsei body behavior was studied. Fish swimming was taped with high speed digital video system and undulatory movement of caudal fin has been drawn. Figure 7 shows body outlines taken from movements of fish swimming of Pangasius sanitwongsei by total length 9 $\mathrm{cm}$. While the amplitude of this wave is very low near the head, it increases significantly near the tail. As it has been shown, Re has obtained in the region of adult fish swimming $\left(10^{3}<\operatorname{Re}<5 \times 10^{6}\right)$ where inertial forces are powerful and viscous forces are neglected. In carangiform and subcarangiform modes, for high speed swimming $\left(10^{4}<\operatorname{Re}<10^{6}\right)$ thrust is optimal for a specific realm of St where $0.25<\mathrm{St}<0.40$ [8]. Figure 9 reveals the relationship between the $\mathrm{A} / \mathrm{L}$ and $\mathrm{Lf} / \mathrm{L}$ of four Pangasius sanitwongsei. $\mathrm{A} / \mathrm{L}$ is the ratio of amplitude to total length. This 


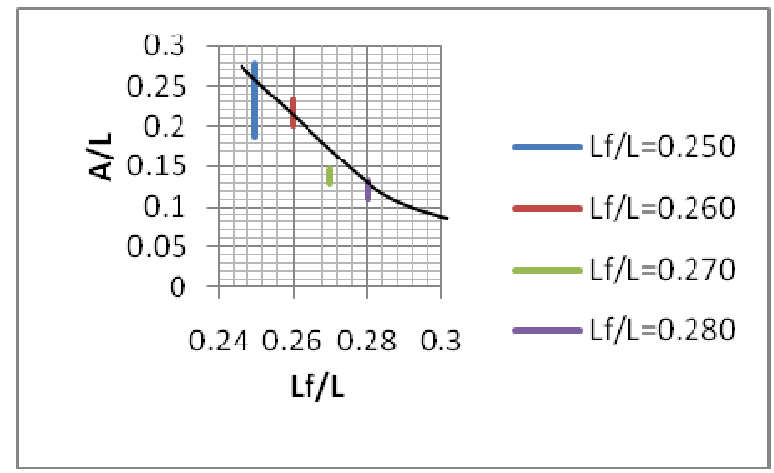

Figure 9: The relationship between $\mathrm{A} / \mathrm{L}$ and $\mathrm{Lf} / \mathrm{L}$.

figure shows a decrease in $\mathrm{A} / \mathrm{L}$ while $\mathrm{Lf} / \mathrm{L}$ increases. In other words, if the ratio of the end part of the fish with high undulatory movement (body and caudal fin) to the total length goes up, the ratio of amplitude to the total length would increase, and consequently thrust force would increase too.

Based on this figure, an experimental equation was found upon which is represented the relationship between $\mathrm{A} / \mathrm{L}$ and $\mathrm{Lf} / \mathrm{L}$ of Pangasius sanitwongsei in steady swimming;

$$
(\mathrm{A} / \mathrm{L})=\mathrm{p} \cdot(\mathrm{Lf} / \mathrm{L})^{\mathrm{k}}
$$

where, $\mathrm{k}=-7.109$ and $\mathrm{p}=1.464$. $\mathrm{k}$ and $\mathrm{p}$ have been named I.A. coefficient.

Fish swimming was described using a traveling wave [14]:

$$
\mathrm{y}_{\text {body }}(\mathrm{x}, \mathrm{t})=\mathrm{a}(\mathrm{x}) \sin (\mathrm{kx}+\omega \mathrm{t})
$$

where, $\mathrm{y}_{\text {body }}$ is the transverse displacement of body and caudal fin, $\mathrm{k}$ is the number of waves; $k=2 \pi / \lambda$ and $\lambda$ is the wave length, $\omega=2 \pi f$ and $f$ is the frequency, $\mathrm{x}$ is the displacement along the main axis and $\mathrm{t}$ is time. Finally $\mathrm{a}(\mathrm{x})$ is the second order function which describes wave amplitude where

$$
\mathrm{a}(\mathrm{x})=\mathrm{c}_{2} \mathrm{x}^{2}+\mathrm{c}_{1} \mathrm{x}+\mathrm{c}_{0} .
$$

In this work, $\mathrm{a}(\mathrm{x})$ for Pangasius sanitwongsei was calculated in steady swimming by experimental trial. Then fish swimming was observed and taped. According to the video images, pattern of body undulatory movement of Pangasius sanitwongsei by $\mathrm{L}=9 \mathrm{~cm}$ were drawn (Fig. 7).

As it was described earlier, undulatory movement of Pangasius sanitwongsei was studied, where frequency of body-caudal fin and wave length were $8.33 \mathrm{1} / \mathrm{s}$ and $0.092 \mathrm{~m}$ respectively. Fish swimming was studied at four sequences at intervals of $20 \mathrm{~ms}$ and movement trajectories were found. Figure 10 shows pattern of body-caudal fins undulatory movement of Pangasius sanitwongsei by $\mathrm{L}=9 \mathrm{~cm}$ at different times. The fish swims at constant velocity, $\mathrm{u}=0.48 \mathrm{~m} / \mathrm{s}$. 


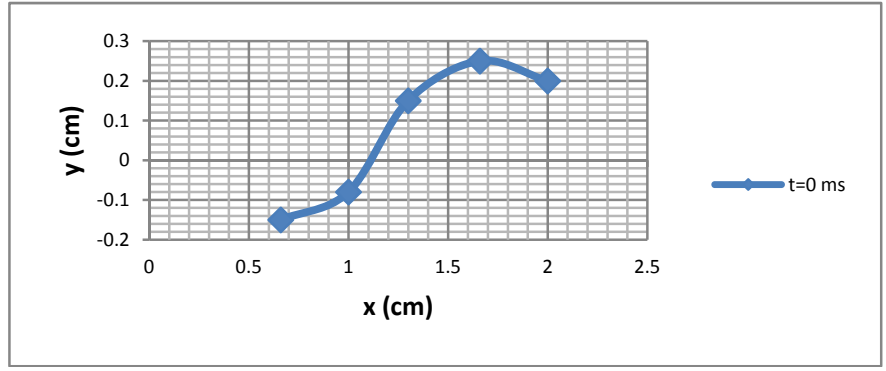

(a)

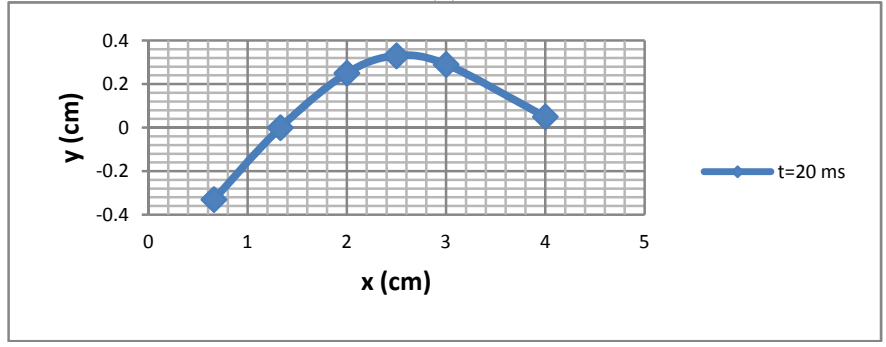

(b)

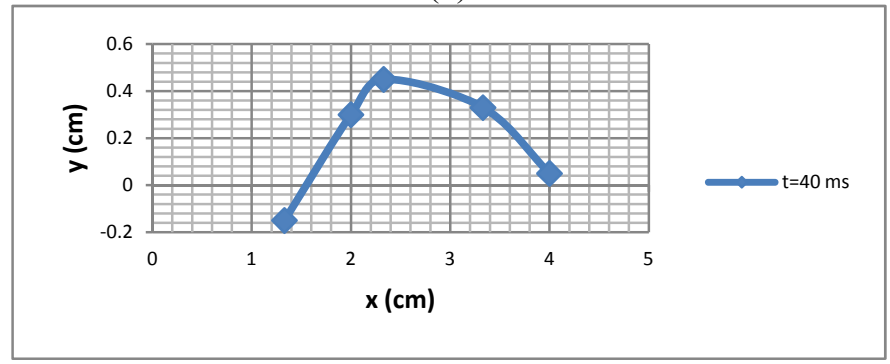

(c)

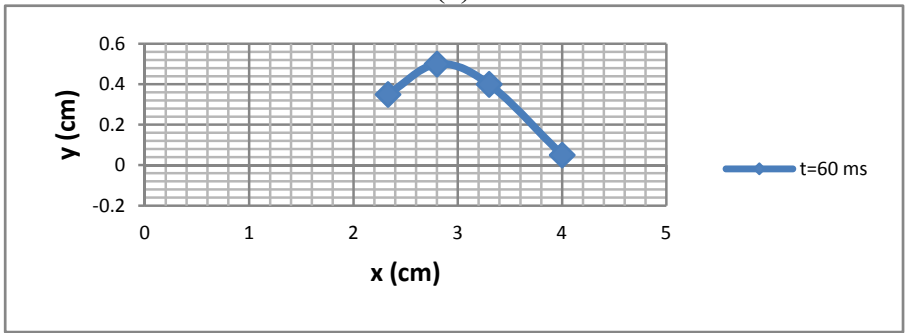

(d)

Figure 10: Pattern of body-caudal fins undulatory movement of Pangasius sanitwongsei by $\mathrm{L}=9 \mathrm{~cm}$ at four sequence at intervals of $20 \mathrm{~m} \mathrm{sec}$. Fish swims at constant velocity, $\mathrm{u}=0.48 \mathrm{~m} / \mathrm{s}$.

To find the time-averaged movement equation, all patterns of body-caudal fins undulatory movement of Pangasius sanitwongsei by $\mathrm{L}=9 \mathrm{~cm}$, were put together in Figure 11. Then a second order function was fitted using second 
order regression which can describe wave-like motion amplitude. Therefore, the generated equation in this work describes the time-averaged movement pattern of caudal fins;

$$
y=-0.1641 x^{2}+0.8774 x-0.8035
$$

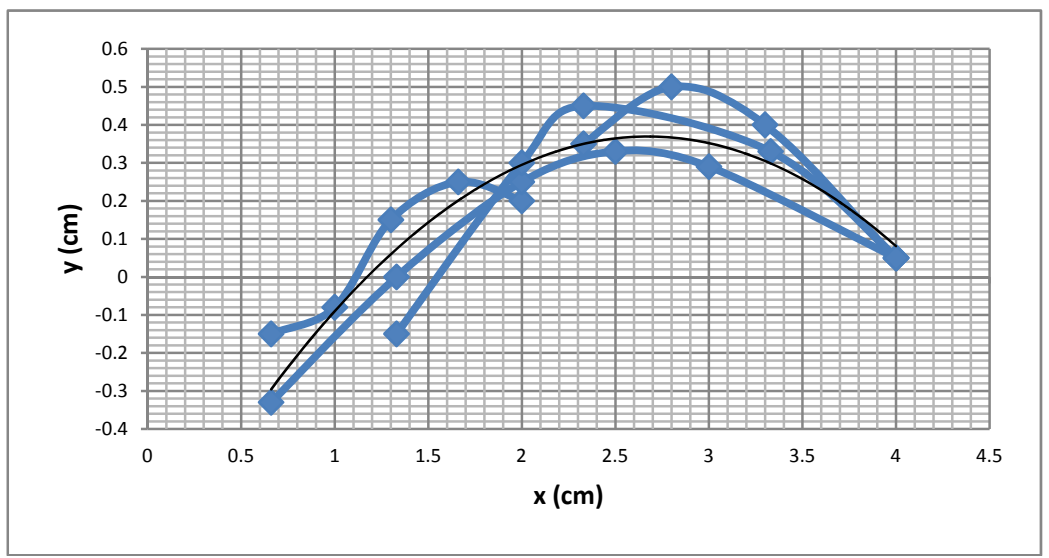

Figure 11: All patterns of body-caudal fins undulatory movement of Pangasius sanitwongsei by $\mathrm{L}=9 \mathrm{~cm}$. time-averaged movement pattern has been presented here.

\section{Conclusions and future directions}

In this paper, kinematic parameters in steady swimming of Pangasius sanitwongsei were studied and patterns of body undulatory movement were extracted. Recording the fish swimming, this experimental study reveals that while the amplitude of the wave near the head of fish is low, it dramatically increases near its tail. Moreover, as the results show the undulatory movement of caudal fin of Pangasius sanitwongsei is almost similar to a sinusoidal form, particularly when $\mathrm{Lf} / \mathrm{L}$ increases. This study resulted in an experimental equation which signifies the relationship between $\mathrm{A} / \mathrm{L}$ and $\mathrm{Lf} / \mathrm{L}$ of Pangasius sanitwongsei in steady swimming. It is concluded that in fish with high undulatory movement (body and caudal fin), increasing in the ratio of the end part of the total length would lead to an increase in the ratio of amplitude to the total length. In other words, while Lf/L goes down, A/L grows up, and thus, both thrust force and displacement increase. It seems that these results are also applicable to robotic fish. Therefore, $\mathrm{Lf} / \mathrm{L}$ is an indication for finding the joint point of robotic fish.

Also, the movement equation of fish swimming was calculated and a second order function describing wave amplitude of Pangasius sanitwongsei was found. This equation works as a function of a real fish and enables us to design a robotic fish by simulating this equation with servo motors at the joint points.

These relations can be applied for mathematical modeling of robot fish. Based on these findings, a robotic fish is being made in the marine engineering 
laboratory at SRBIAU (Science and Research Branch, Islamic Azad University). Finally, it is suggested for future studies to follow numerical evaluations based on these findings.

\section{References}

[1] Arakeri, J.H., Fluid Mechanics of Fish Swimming. Resonance, 14(1), pp. 32-46, 2009.

[2] Hu, H., Liu, J., Dukes, I. and Francis, G., Design of 3d swim pattern for autonomous robotic fish. IEEE., International Conference on Intelligent Robots and Systems, pp. 2406-2411, 2006.

[3] Streitlien, G.S. and Triantafyllou, M.S., Efficient foil propulsion through vortex control. AIAA Journal., 34(11), pp. 2315-2319, 1996.

[4] Anderson, J. M., Streitlien, K., Barrett, D. S and Triantafyllou, M. S, Oscillating foils of high propulsive efficiency. J. Fluid Mech. 360, pp. 41$72,1998$.

[5] Gillbert, D., Quantitative characterization of three-dimensional pectoral fin kinematics in Bluegill sunfish, Lepomis macrochirus., Murj J. 10, pp. 5864, 2004.

[6] Lauder, G.V., Nauen, J.C., and Drucker, E.G., Experimental hydrodynamics and evolution: function of median fins in ray- finned fishes. I. \& C. Biology, 42(5), pp. 1009- 1017, 2002.

[7] Standen, E.M and Lauder G.V., Hydrodynamic function of dorsal and anal fins in brook trout (Salvelinusfontinalis). The Journal of Experimental Biology, 210, pp. 325-339, 2007.

[8] Stafiotakis, M., Lane, D. M., and Davies, J. B. C., Review of fish swimming modes for aquatic locomotion. IEEE, J. Oceanic engineering, 24(2), pp. 237-252, 1999.

[9] Lauder, G.V. and Drucker, E.G., Morphology and experimental hydrodynamics of fish Fin control surfaces. IEEE, J. Oceanic engineering, 29(3), pp. 556-571, 2004.

[10] Lindsey C.C. Form function and locomotory habits in fish. Fish physiology. Locomotion, New York Academic, VII, pp. 1-100, 1987.

[11] Webb, P. W., Simple physical principles and vertebrate aquatic locomotion. American zoologist. 28, pp. 709-725, 1988.

[12] Takeuchi, S., Kusada, S. and Kajishima, T., Optimisation of fish shape and Swim mode in fully resolved 2-D flow field by genetic algorithm with the least square prediction method. (Chapter13). Bio-mechanisms of Swimming and Flying, ed. N. Kato and S. Kamimura, Springer-Verlag: Tokyo, pp. 155-166, 2007.

[13] Triantafillou, G.S., Triantafillou, M.S. and Grosenbauch, M.A, Optimal thrust development in oscillating foils with application to fish propulsion. J. Fluid Struct., 7, pp. 205-224, 1993.

[14] Liu, J. and $\mathrm{Hu}, \mathrm{H}$., A 3D simulator for autonomous robotic fish. International Journal of Automation and Computing, 1, pp. 42-50, 2004. 